\title{
Fundamental mechanisms responsible for the temperature coefficient of resonant frequency in microwave dielectric ceramics
}

\author{
Shengke Zhang ${ }^{1}$ | Hasan Sahin ${ }^{2,4}$ | Engin Torun' ${ }^{2}$ Francois Peeters ${ }^{2}$ | Dinesh Martien ${ }^{3}$ | \\ Tyler DaPron $^{3}$ | Neil Dilley ${ }^{3}$ | Nathan Newman ${ }^{1}$
}

${ }^{1}$ Arizona State University, Tempe,

Arizona

${ }^{2}$ Department of Physics, University of Antwerp, Antwerp, Belgium

${ }^{3}$ Quantum Design, San Diego, California

${ }^{4}$ Department of Photonics, Izmir Institute of Technology, Izmir, Turkey

Correspondence

Nathan Newman, Arizona State

University, Tempe, AZ.

Email: Nathan.Newman@asu.edu

\begin{abstract}
The temperature coefficient of resonant frequency $\left(\tau_{\mathrm{f}}\right)$ of a microwave resonator is determined by three materials parameters according to the following equation: $\tau_{\mathrm{f}}=-\left(1 / 2 \tau_{\varepsilon}+1 / 2 \tau_{\mu}+\alpha_{\mathrm{L}}\right)$, where $\alpha_{\mathrm{L}}, \tau_{\varepsilon}$, and $\tau_{\mu}$ are defined as the linear temperature coefficients of the lattice constant, dielectric constant, and magnetic permeability, respectively. We have experimentally determined each of these parameters for $\mathrm{Ba}$ $\left(\mathrm{Zn}_{1 / 3} \mathrm{Ta}_{2 / 3}\right) \mathrm{O}_{3}, 0.8$ at.\% Ni-doped $\mathrm{Ba}\left(\mathrm{Zn}_{1 / 3} \mathrm{Ta}_{2 / 3}\right) \mathrm{O}_{3}$, and $\mathrm{Ba}\left(\mathrm{Ni}_{1 / 3} \mathrm{Ta}_{2 / 3}\right) \mathrm{O}_{3}$ ceramics. These results, in combination with density functional theory calculations, have allowed us to develop a much improved understanding of the fundamental physical mechanisms responsible for the temperature coefficient of resonant frequency, $\tau_{\mathrm{f}}$.
\end{abstract}

\section{K E Y W O R D S}

density functional theory, dilatation/dilatometry, dopants/doping, electron spin resonance, microwave resonators

\section{1 | INTRODUCTION}

Low-loss temperature-compensated $\mathrm{Ba}\left(\mathrm{B}_{1 / 3}^{\prime} \mathrm{Ta}_{2 / 3}\right) \mathrm{O}_{3} \quad\left(\mathrm{~B}^{\prime}=\right.$ $\left.\mathrm{Zn}^{2+}, \mathrm{Nb}^{2+}, \mathrm{Mg}^{2+}\right)$ perovskite dielectrics are used extensively in high-performance communication and Doppler systems. Of these, cellular base station filters have become the most common application. ${ }^{1,2}$ Such advanced microwave systems require that the relative temperature dependence of the dielectric's resonant frequency, $\tau_{\mathrm{f}}=\left({ }^{1} / f\right)\left({ }^{\mathrm{d} f} / \mathrm{d} T\right)$, be precisely set at zero or near-zero to achieve the required performance over a range of temperatures. ${ }^{3}$ A small non-zero $\tau_{\mathrm{f}}$ is often chosen by system engineers to offset the small temperature dependence of the amplifiers. Despite the importance of $\tau_{\mathrm{f}}$ for these practical applications, a strong first-principles understanding of what determines this important parameter has not been established. The goal of this work was to demonstrate that currently available density functional theory (DFT) calculations can be used to

Corrections added on 16 March 2017, after first online publication quantitatively model the physical processes involved in determining $\tau_{\mathrm{f}}$ with reasonable accuracy.

Controlling $\tau_{\mathrm{f}}$ to the desired value in low-loss materials has been achieved empirically by researchers and commercial manufactures by (1) doping, (2) alloying, and/or (3) combining multiphase composite ceramic materials. ${ }^{4-10}$ Surprisingly, at least to the authors of this manuscript, almost all of the commercial microwave dielectric products on the market today tune $\tau_{\mathrm{f}}$ through the addition of varying amounts of magnetic additives, such as $\mathrm{Ni}, \mathrm{Co}$, and $\mathrm{Mn}^{6-}$ ${ }^{10}$ In this work, $\mathrm{Ba}\left(\mathrm{Zn}_{1 / 3} \mathrm{Ta}_{2 / 3}\right) \mathrm{O}_{3}$ (BZT) was selected for the host material because it is the lowest-loss temperaturecompensated microwave ceramic with a dielectric constant of $\sim 30$ or greater. $0.8 \% \mathrm{Ni}$-doping was used since this concentration of $\mathrm{Ni}$ doping in commercial materials tunes $\tau_{\mathrm{f}}$ to near-zero. ${ }^{8,11}$ Finally, since the $\mathrm{Ba}\left(\mathrm{Zn}_{1 / 3-x} \mathrm{Ni}_{x} \mathrm{Ta}_{2 / 3}\right) \mathrm{O}_{3}$ system is a completely miscible solid solution whose roomtemperature dielectric constant and $\tau_{\mathrm{f}}$ vary smoothly and monotonically over the entire alloy series, we believe that the inferences we derive from 0.8 at. $\%$ and $\mathrm{Ba}\left(\mathrm{Ni}_{1 / 3} \mathrm{Ta}_{2 / 3}\right)$ 
$\mathrm{O}_{3}(\mathrm{BNT})$ are general and can be used to understand other concentrations. ${ }^{6,9,10}$

To develop a strong first-principles understanding, we first must determine how material properties are related to this important device parameter, $\tau_{\mathrm{f}}$. The resonant frequency, $f$, for a representative structure, such as a half-wavelength resonator of length $l$, is $\frac{c}{2 l \sqrt{\varepsilon \mu}}$ where $c$ is the speed of light under vacuum, $\varepsilon$ is the dielectric constant, and $\mu$ is the magnetic permeability. ${ }^{3}$ Then by taking partial derivatives with respect to temperature, we obtain the more general equations.

$$
\begin{gathered}
\frac{\mathrm{df}}{\mathrm{dT}}=-\frac{\mathrm{c}}{2 \mathrm{l} \sqrt{\varepsilon \mu}} \times \frac{\mathrm{dl}}{\mathrm{ldT}}-\frac{\mathrm{c}}{4 \mathrm{l} \sqrt{\varepsilon \mu}} \times \frac{\mathrm{d} \varepsilon}{\varepsilon \mathrm{dT}}-\frac{\mathrm{c}}{4 \mathrm{l} \sqrt{\varepsilon \mu}} \times \frac{\mathrm{d} \mu}{\mu \mathrm{dT}} \\
=-\mathrm{f} \times \frac{\mathrm{dl}}{\mathrm{ldT}}-\frac{\mathrm{f}}{2} \times \frac{\mathrm{d} \varepsilon}{\varepsilon \mathrm{dT}}-\frac{\mathrm{f}}{2} \times \frac{\mathrm{d} \mu}{\mu \mathrm{dT}} \\
\frac{\mathrm{df}}{\mathrm{fdT}}=-\frac{\mathrm{dl}}{\mathrm{ldT}}-\frac{1}{2} \times \frac{\mathrm{d} \varepsilon}{\varepsilon \mathrm{dT}}-\frac{1}{2} \times \frac{\mathrm{d} \mu}{\mu \mathrm{dT}}
\end{gathered}
$$

Thus,

$$
\tau_{f}=-\left(\alpha_{L}+\frac{1}{2} \tau_{\epsilon}+\frac{1}{2} \tau_{\mu}\right)
$$

The three material parameters, $\alpha_{\mathrm{L}}=(1 / l)(\mathrm{d} l / \mathrm{d} T), \quad \tau_{\varepsilon}=(1 / \varepsilon)$ $\left({ }^{\mathrm{d} \varepsilon} / \mathrm{d} T\right)$, and $\tau_{\mu}=(1 / \mu)\left({ }^{\mathrm{d} \mu} / \mathrm{d} T\right)$ in Equation (3), are referred to the linear temperature coefficients of the lattice constant (i.e., thermal expansion coefficient), dielectric constant, and magnetic permeability, respectively. ${ }^{1,2}$

To better understand the mechanism responsible for these three parameters, it is useful to establish their temperature dependences from near $0 \mathrm{~K}$, where thermal excitations of phonon, electronic and spin excitations are minimal, up to say their operating temperature of $\sim 300 \mathrm{~K}$. We are not aware of any published papers in the literature that have determined all the relevant parameters, $\tau_{\varepsilon}, \tau_{\mu}, \alpha_{L}$, and $\tau_{\mathrm{f}}$, for a microwave ceramic material at cryogenic temperatures. In the next few paragraphs we will summarize the literature that investigated the material's properties of microwave perovskite's at low temperature.

There have been a handful of studies ${ }^{12-15}$ which have characterized only the parameter $\alpha_{\mathrm{L}}$ for low-loss perovskite ceramics over this temperature range. In an investigation of the thermal expansion of $\mathrm{Ba}\left(\mathrm{Mg}_{1 / 3} \mathrm{Ta}_{2 / 3}\right) \mathrm{O}_{3}\left(\tau_{\mathrm{f}} @ 300 \mathrm{~K}=\right.$ $5.4 \mathrm{ppm} / \mathrm{K})$, Gvasaliya et al. $^{13}$ reported that the degree of $\mathrm{Mg}^{2+} / \mathrm{Ta}^{5+}$ does not significantly influence the material's thermal expansion. $\alpha_{\mathrm{L}}$ of $\mathrm{BaZrO}_{3}\left(\tau_{\mathrm{f}} @ 300 \mathrm{~K}=\sim 140 \mathrm{ppm} / \mathrm{K}\right)$ was measured by neutron scattering at three temperatures between 2 and $300 \mathrm{~K},{ }^{14,15}$ making it difficult to observe systematic trends.

A number of other studies have measured $\tau_{f}$ using a $\mathrm{TE}_{01 \delta}$ or $\mathrm{TE}_{011}$ resonance mode of dielectric resonators ${ }^{16-22}$ and assumed that this is equal to $\tau_{\varepsilon}$, neglecting the contributions from $\alpha_{\mathrm{L}}$ and $\tau_{\mathrm{u}}$. From the literature ${ }^{1,23}$ and the measurements reported here, we note that $\alpha_{L}$ can be on the order of 5-10 ppm/K at $200 \mathrm{~K}$ and above for most microwave oxide ceramics, ${ }^{1}$ whereas $\tau_{\mathrm{u}}$ can be of the same order at very low temperature in paramagnetic-laden dielectrics. ${ }^{24}$ Since these References ${ }^{16-22}$ directly measured $\tau_{\mathrm{f}}$, we will only summarize their findings in that light. Subodh et al. ${ }^{19}$ found that as the temperature increases, the $\tau_{\mathrm{f}}$ of $\mathrm{V}_{2} \mathrm{O}_{5^{-}}$ doped $\mathrm{Mg}_{2} \mathrm{TiO}_{4}$ dielectrics is $\sim-30 \mathrm{ppm} / \mathrm{K}$ at low temperatures and increases to $\sim-50 \mathrm{ppm} / \mathrm{K}$ above $150 \mathrm{~K}$. An investigation of $\mathrm{V}_{2} \mathrm{O}_{5}$-doped $\mathrm{Mg}_{2} \mathrm{TiO}_{4}$ ceramics by Bhuyan et al. ${ }^{16}$ and single-crystal $\mathrm{LaAlO}_{3}{ }^{20}$ found that $\tau_{\mathrm{f}}$ is positive at low temperature, increasing slowly as the temperature is raised to $\sim 150-200 \mathrm{~K}$, then increasing more rapidly above 150-200 K. Similarly, Pamu et al. found that $\tau_{\mathrm{f}}$ of $\mathrm{CuO}-$ doped $\mathrm{Zr}_{0.8} \mathrm{Sn}_{0.2} \mathrm{TiO}_{4}$ is $\sim 9 \mathrm{ppm} / \mathrm{K}$ from 10 to $250 \mathrm{~K}$ and then increases significantly up to room temperature. ${ }^{21}$ Likewise, Varma et al. reported that 0.5 at.\% of a number of dopants including In, Ti, Mn, and $\mathrm{Sb}$ in $\mathrm{Ba}\left(\mathrm{Zn}_{1 / 3} \mathrm{Ta}_{2 / 3}\right) \mathrm{O}_{3}$ ceramics have a $\tau_{\mathrm{f}}$, of $\sim-5$ at $25 \mathrm{~K}$ that rises to +2 to $5 \mathrm{ppm} / \mathrm{K}$ at room temperature. Of these dopants in $\mathrm{Ba}\left(\mathrm{Zn}_{1 / 3} \mathrm{Ta}_{2 / 3}\right) \mathrm{O}_{3}$, only the Ti dopant shows any substantial temperature dependence in the range from 200 to $300 \mathrm{~K}^{22}$ In that study, higher doping levels were found to exhibit similar increases in $\tau_{\mathrm{f}}$ with temperature, but with quantitatively different values over the entire range of temperatures. In contrast, Jacob et al. ${ }^{17}$ found that $\tau_{\mathrm{f}}$ of $\mathrm{CuO} / \mathrm{ZnO}$ doped $\mathrm{Zr}_{0.8} \mathrm{Sn}_{0.2} \mathrm{TiO}_{4}$ is essentially constant at $\sim 5 \mathrm{ppm} / \mathrm{K}$ from 13 to $300 \mathrm{~K}$. Interestingly, Sudheendran et al. ${ }^{18}$ presented that at cryogenic temperatures, $\tau_{\mathrm{f}}$ of 0.9 at. $\% \mathrm{Ti}^{4+}$-doped $\mathrm{Bi}_{2}\left(\mathrm{Zn}_{2 / 3} \mathrm{Nb}_{4 / 3}\right) \mathrm{O}_{7}$ could be negative from $\sim 10$ to $75 \mathrm{~K}$; then it becomes positive and almost constant at the higher temperatures. In an early investigation, Tamura also observed that $\tau_{\mathrm{f}}$ was nonmonotonic with temperature. ${ }^{25}$ From this, it is clear that the temperature dependence of $\tau_{f}$ varies widely and an improved understanding of the mechanisms that determine this important property is needed.

In our study, we have experimentally measured $\alpha_{\mathrm{L}}, \tau_{\mathrm{f}}$, and $\tau_{\mu}$ of BZT, 0.8 at.\% Ni-doped BZT and $\mathrm{Ba}\left(\mathrm{Ni}_{1 / 3} \mathrm{Ta}_{2 / 3}\right)$ $\mathrm{O}_{3}$ (BNT), allowing us to determine $\tau_{\varepsilon}$ over the temperature range 2-300 K. We also performed first-principles calculations for the electronic and phonon structures of the compounds. This has allowed us to gain a much improved fundamental quantitative understanding of the mechanisms responsible for the observed temperature dependence of these parameters

\section{2 | EXPERIMENTAL PROCEDURE}

$\mathrm{Ba}\left(\mathrm{Zn}_{1 / 3} \mathrm{Ta}_{2 / 3}\right) \mathrm{O}_{3} \quad$ (BZT), $\mathrm{Ba}\left(\mathrm{Ni}_{1 / 3} \mathrm{Ta}_{2 / 3}\right) \mathrm{O}_{3} \quad$ (BNT), and 0.8 at.\% Ni-doped BZT dielectrics studied in this work were synthesized by conventional ceramic powder processing methods in our laboratory. ${ }^{23}$ The resonant frequencies were determined using microwave dielectric resonator (DR) 
$1510 \mid \bigcirc 0$

measurements. The $0.85 \mathrm{~cm} \times 0.60 \mathrm{~cm} \times 0.20 \mathrm{~cm}$ rectangular DRs were inserted into a $2.03 \mathrm{~cm} \times 0.61 \mathrm{~cm} \times 1.52 \mathrm{~cm}$ gold-plated copper cavity which was affixed to the end of a cryogenic dipping probe. A HP8510C microwave vector network analyzer (HewlettPackard, Palo Alto, CA) was used to measure $S_{21}$ vector transmission values. Micrometers are used to adjust the distance between the microwave coupling probes and sample that is weakly coupled to the resonator, allowing the unloaded $Q$ of the DR to be determined to better than a few percent and the corresponding resonant frequency to be determined to typically better than $5 \mathrm{ppm}$. The microwave cavity was lowered into a cryostat (PPMS, Model 6000; Quantum Design, San Diego, CA) and the resonant frequency of the $\mathrm{TE}_{11 \delta}$ mode of rectangular DRs was measured in the range 9.5-10.0 GHz. ${ }^{23,24}$ Static dielectric constants, $\varepsilon_{0}$, were measured by a conventional $\mathrm{TE}_{01 \delta}$ measurements technique on $\sim 2.7 \mathrm{~cm}$ diameter, $\sim 0.5 \mathrm{~cm}$ tall cylindrical samples in an $8.0 \mathrm{~cm}$ diameter $\times 7.0$ high cylindrical gold-plated copper cavity at room temperature. ${ }^{23,24}$

Magnetic permeability determinations of $\mu=1+\mathrm{M} / \mathrm{H}$ are performed using a vibrating sample magnetometer option for the Quantum Design PPMS system (Model 6000; Quantum Design, San Diego, CA).

For this study, we used a commercial direct-reading capacitance dilatometer from Quantum Design (Model PPMS with the dilatometer option). This design directly infers temperature-induced changes in the sample length from the reactance difference between two capacitors in the dilatometer cell. In this regard, an absolute capacitance measurement is not required and the resulting symmetrical configuration reduces the effects of absorbed gasses and temperature gradients. The cell is constructed from fused silica, resulting in a low thermal expansion background.

These methods were chosen to determine $\tau_{\mathrm{f}}, \tau_{\varepsilon}, \tau_{\mu}$, and $\alpha_{L}$ with the required precision of better than a ppm. ${ }^{26,27}$ Ideally, a direct method to determine the dielectric constant as a function of temperature would be preferred, but unfortunately the established methods for this, such as the coaxial probe, transmission line, or free space transmission or reflection techniques, ${ }^{28}$ are only accurate to a few percent under typical measurement conditions and to a little better than a part per thousand under the most optimal laboratory conditions. ${ }^{26,27}$ Nonresonant indirect (device) techniques, such as measuring the capacitance in the parallel plate geometry, do not have any better precision than a part per thousand too and would also need to be corrected for lattice expansion. ${ }^{26-28}$ Thus, neither the direct methods nor nonresonant indirect methods offer the precision needed a few $\mathrm{ppm} / \mathrm{K}$ needed for this study. So, we used an electromagnetic resonator technique with the required precision to infer $\tau_{\varepsilon}$ by correcting the measured temperature-dependent frequency shifts $\left(\tau_{f}\right)$ with suitably accurate measurements of the lattice expansion $\left(\alpha_{\mathrm{L}}\right)$ and the temperature dependence of magnetic permeability $\left(\tau_{\mu}\right)$ (i.e., Equation 3 ).

The DFT calculations were performed on BZT and BNT crystal structures using plane wave projector-augmented wave method ${ }^{29}$ which is implemented in the Vienna Ab-initio Simulation Package ${ }^{30-32}$ The generalized gradient approximation within the Perdew-Burke-Ernzerhof ${ }^{33}$ is employed to describe the exchange-correlation potential. The kinetic energy cutoff for plane-wave expansion is set to $500 \mathrm{eV}$ and the hexagonal Brillouin Zone was sampled using $14 \times 14 \times 12 \Gamma$ centered k-point grid for the primitive cell and for the examination of magnetic ground state it is scaled according to size of the supercell. The convergence criterion for energy is set to $10^{-5} \mathrm{eV}$ between two consecutive steps in the self-consistent field calculations. Full structural relaxation was performed until the Hellmann-Feynman forces are less than $10^{-4} \mathrm{eV} / \AA$. Phonon calculations were performed using small displacement methodology implemented in the PHON code. ${ }^{34}$ The imaginary part of the electronic contribution of the dielectric function of BZT and BNT is calculated by summing over empty electronic states using Equation (4).

$$
\begin{aligned}
\varepsilon_{\alpha \beta}(w) & =\frac{4 \pi^{2} e^{2}}{\Omega} \lim _{q \rightarrow 0} \frac{1}{q^{2}} \sum_{c, v, k} 2 w_{k} \delta\left(\epsilon_{c k}-\epsilon_{v k}-w\right) \\
& \times\left\langle u_{c k+e_{\alpha q}}\left|u_{v k} u_{v k}\right| u_{c k+e_{\beta q}}\right\rangle
\end{aligned}
$$

where $q, \Omega, \varepsilon, w, u_{c k}, c$, and $v$ correspond to Bloch vector, volume of the unit cell, dielectric function, frequency, cell periodic part of the orbitals at the $k$-point $(k)$, conduction, and valence band states, respectively.

For this work, we only calculated the electronic and phonon band structures of the compounds $\mathrm{Ba}\left(\mathrm{Zn}_{1 / 3} \mathrm{Ta}_{2 / 3}\right) \mathrm{O}_{3}$ and $\mathrm{Ba}\left(\mathrm{Ni}_{1 / 3} \mathrm{Ta}_{2 / 3}\right) \mathrm{O}_{3}$ and did not carry out simulations for the random alloy $\mathrm{Ba}\left(\left[\mathrm{Zn}_{1-x} \mathrm{Ni}_{x}\right]_{1 / 3} \mathrm{Ta}_{2 / 3}\right) \mathrm{O}_{3}$ due to the very expensive computational cost required to accurately simulate a very large number of atoms required for random alloys with exchange interactions.

\section{3 | RESULTS AND DISCUSSION}

In Figure 1, we present our experimental measurements of $\tau_{\mathrm{f}}$, the factor of interest to microwave system designers. Then, in the remainder of the section, we give experimental and simulation results for $\alpha_{L}, \tau_{\varepsilon}$, and $\tau_{\mu}$, parameters that describe the temperature dependence of the physical properties that determine this important factor.

\subsection{Determining $\alpha_{L}$}

Next, we provide experimental determinations of $\alpha_{L}$ and show that it can be quantitatively modeled and understood 


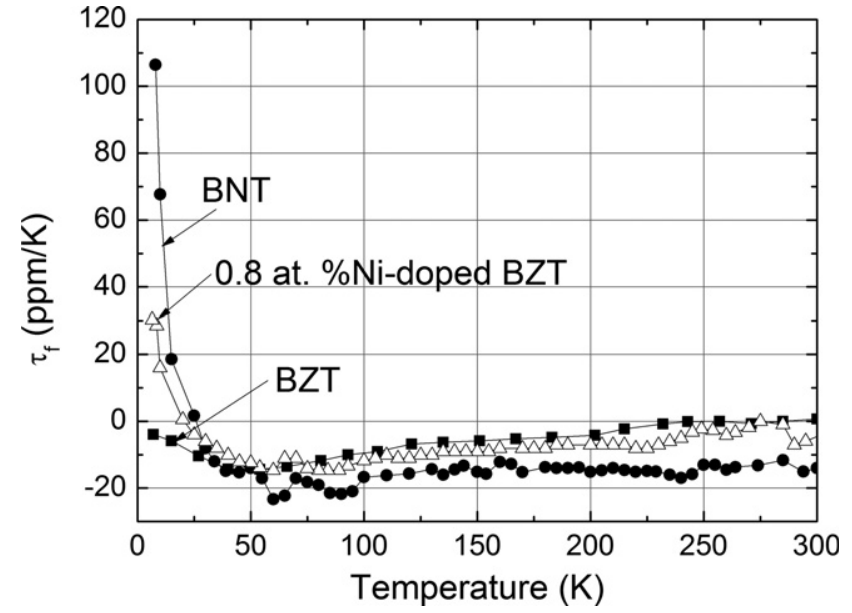

FIGURE 1 Temperature dependence of temperature coefficient of resonant frequency $\left(\tau_{\mathrm{f}}\right)$ in BZT, BNT, and 0.8 at.\% Ni-doped BZT.

using our DFT calculations. In Figure 2A, we see that the thermal BZT and BNT expansion data up to $\sim 200 \mathrm{~K}$ can be accurately fit with the Debye equation for the specific heat and Grüneisen analysis ${ }^{36}$ using a characteristic Debye temperature of 392 and $408 \mathrm{~K}$, respectively. When the temperature is above $200 \mathrm{~K}, \alpha_{\mathrm{L}}$ starts to deviate from the Grüneisen model as a result of the extra weight of softened modes near the Brillion zone boundaries due to an antidistortive transition (Figure 3). It is also noticed that the thermal expansion reduces as the $\mathrm{Ni}$ concentration increases, which is often correlated with the smaller atomic weight and shorter effective ionic radii of the $\mathrm{Ni}^{2+}$ ion than $\mathrm{Zn}^{2+} 38$

The results of the DFT calculations of the phonon dynamics are shown in Figure 3. From the slope of the lowest energy acoustic phonon mode of the simulation, we estimate the Debye temperature of BZT and BNT to be $\sim 438 \mathrm{~K}$ and $\sim 480 \mathrm{~K}$, respectively, $\sim 15 \%$ higher than found when fitting experimental data. This is a reasonable agreement, given that our estimate of the Debye temperature from the theory comes only from only the lowest energy acoustic mode. Both theory and experiment find that BNT has a larger Debye temperature than BZT. Given that Ni has a lighter atomic mass than $\mathrm{Zn}$ by $\sim 10 \%$, we would expect BNT to have a higher sound velocity and thus Debye temperature. Even though simple models indicate that these properties scale with the effective spring constant and inversely with the square root of the atomic mass, it is difficult to make quantitative estimates given that the Debye temperature in these materials is established by many vibrational modes each involving a number of the compound's elemental constituents.

Using Grüneisen analysis, we can infer $\alpha_{\mathrm{L}}$ from the DFT calculations of the heat capacity $\left(C_{\mathrm{v}}\right)$ using Equation (5). Results for $\alpha_{\mathrm{L}}$ as a function of temperature are shown in Figure 2B.

$$
\alpha=\frac{\gamma C_{\mathrm{v}}}{3 B}
$$

where $\gamma$ is the average Grüneisen parameters and $B$ is the bulk modulus. ${ }^{30}$ We calculate the Grüneisen parameter $(\gamma)$ of BZT and BNT using, $\gamma=-\frac{\operatorname{dn}(\omega)}{\operatorname{dn}(\mathrm{V})}$ where $V$ and $\omega$ correspond to volume of the unit cell and the average Debye frequency of the acoustic modes. The Grüneisen parameter of BZT and BNT are calculated to be 2.39 and 1.66, respectively. This analysis indicates that small thermal expansion coefficients are expected from compressible
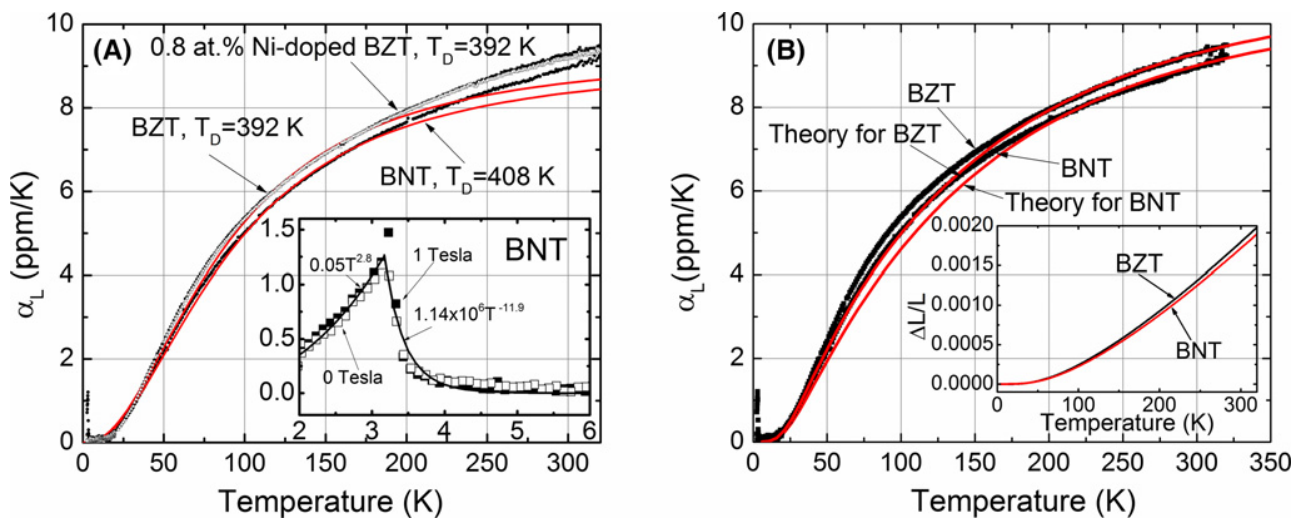

FIGURE 2 A, Thermal expansion $\left(\alpha_{\mathrm{L}}\right)$ measurement values for BZT and BNT are represented as black data points and the Grüneisen model fits are represented as red solid lines. The inset shows BNT's Neel temperature at $3.3 \mathrm{~K}$, characteristic of a phase transition from the antiferromagnetic state at low temperatures to the paramagnetic state. ${ }^{35}$ A peak in the BNT magnetic permeability at $3.3 \mathrm{~K}$ (inset of Figure $4 \mathrm{~B}$ ) confirms this. Neither BZT or 0.8 at.\% Ni-doped BZT show evidence for a peak in their thermal expansion characteristics, indicating that they are not antiferromagnetic over the measured temperature range. $\mathrm{B}, \alpha_{\mathrm{L}}$ (black data points) and density functional theory results (red solid lines) of BZT and BNT. The inset shows the temperature dependence of lattice expansion of BZT and BNT, as derived from the integration of the experimental $\alpha_{\mathrm{L}}$. [Color figure can be viewed at wileyonlinelibrary.com] 
lattices whose high-energy phonons do not significantly soften upon expansion (i.e., small Grüneisen parameters). Thus, materials with high Debye temperatures within a given class of material will tend to have smaller thermal expansion coefficients, as we found when comparing the results for $\mathrm{BZT}$ to $\mathrm{BNT}$.

The values of $B_{\mathrm{BZT}}=209 \mathrm{GPa}$ and $\mathrm{B}_{\mathrm{BNT}}=152 \mathrm{GPa}$ were inferred by scaling the theory relation to the experiment values in Figure 2B. We note that the theoretical results closely follow the measurement values, particularly below $50 \mathrm{~K}$ and above $175 \mathrm{~K}$. These observations again confirm that the theoretical calculations can adequately model the phonon dynamics in these materials.

We also used the theory alone to model the bulk modulus of BZT and BNT. We changed the volume of the unit cell from $-2 \%$ to $2 \%$ with step of $1 \%$ and obtained the energy $(E)$ as a function of volume $(V)$. Then, a second order polynomial function was used to fit the $E$ vs $V$ data, and the bulk moduli of BZT and BNT were calculated by taking the second order derivative of $E$ with respect to the $V$, as indicated by Equation (6).

$$
B=V \frac{\mathrm{d}^{2} E}{\mathrm{~d}^{2} V}
$$

where $B$ and $E$ correspond to the bulk modulus and energy, respectively. Our calculations show that the bulk moduli of BZT and BNT are 138.10 and $138.75 \mathrm{GPa}$, respectively. These values are significantly different than inferred from the Grüneisen analysis of the experimental data reported earlier in this section. The variance may arise as a result of using an average Grüneisen parameter rather than
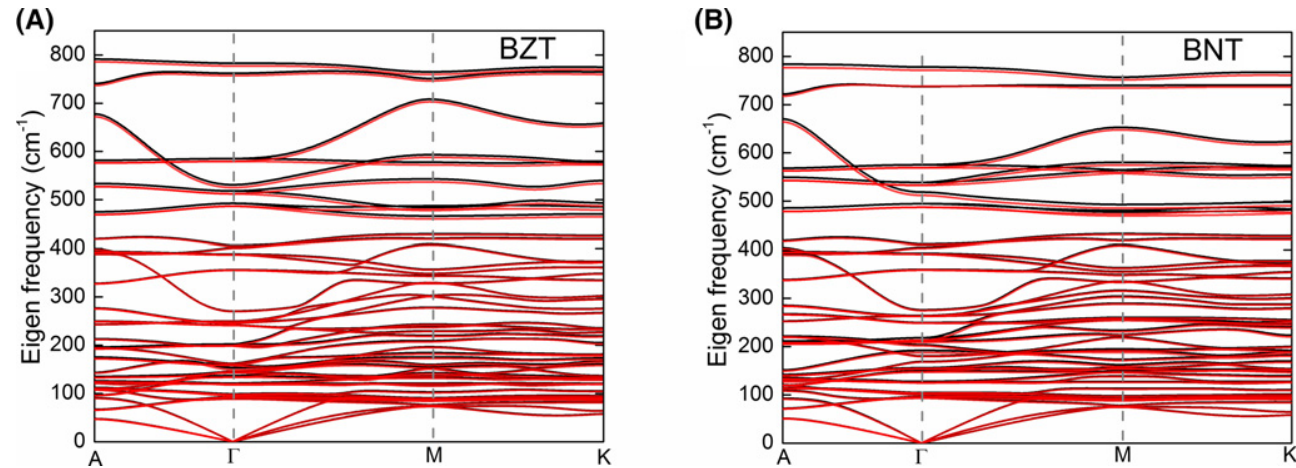

F IGURE 3 Phonon Eigen frequency $(\omega=E / h)$ vs wave vector $(k)$ curves of BZT (A) and BNT (B) with relaxed lattice at equilibrium and at $0 \mathrm{~K}$ (black lines) and at a $0.2 \%$ lattice enlargement (red lines), the experimentally measured increase at $\sim 300 \mathrm{~K}$ (inset Figure $2 \mathrm{~B}$ ). The $0.2 \%$ expanded lattice is used to simulate the properties at $300 \mathrm{~K}$. This is based on the concept that a solid's properties can be adequately modeled by

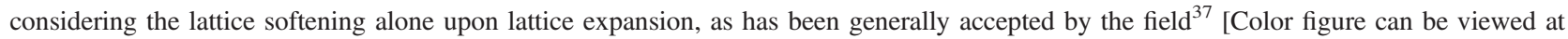
wileyonlinelibrary.com]
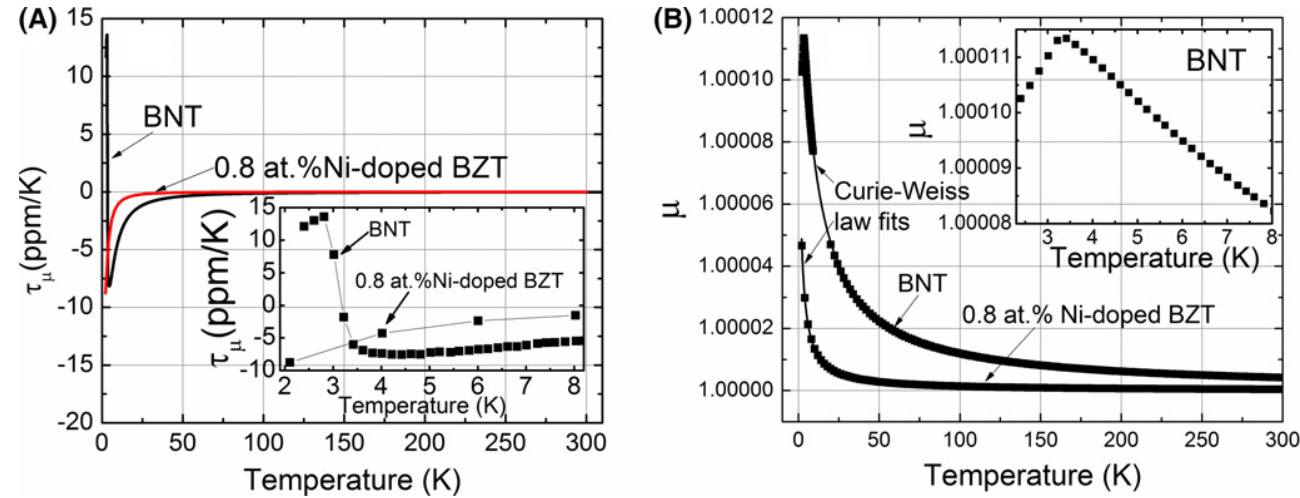

F I G URE 4 (A) Temperature coefficient of magnetic permeability $\left(\tau_{\mu}\right.$, ) of BNT and 0.8 at.\% Ni-doped BZT characterized measured using a vibrating sample magnetometer at $40 \mathrm{~Hz}$. The inset shows the $\tau_{\mu}$ from 2 to $8 \mathrm{~K}$; (B) temperature dependence of permeability ( $\mu$ ) of BNT and 0.8 at.\% Ni-doped BZT, and black solid lines are the Curie-Weiss law fits. The inset shows the antiferromagnetic Neel temperature of BNT is $3.3 \mathrm{~K}$ [Color figure can be viewed at wileyonlinelibrary.com] 
performing a $k$-dependent analysis for every phonon mode. More detailed analysis will be reported in the future.

\section{2 | Determining $\tau_{\mu}$}

Next we show the temperature dependence of permeability ( $\mu$ ) and its temperature-derivative, $\tau_{\mu}$, in Figure 4. Note that the $\mu$ of BNT follows the expected Curie-Weiss law in Equations (7) and (8). ${ }^{36}$

$$
\begin{gathered}
\mu=\frac{C}{(T+\Theta)} \\
\tau_{\mu}=-\frac{C}{(T+\Theta)^{2}}
\end{gathered}
$$

where $\Theta$ is Weiss temperature and $C$ is the Curie constant. The $\Theta$ of BNT is calculated to be $-8.37 \mathrm{~K}$. When the temperature is below the Neel temperature, $3.3 \mathrm{~K}$, the BNT transitioned to antiferromagnetic phase, which shows a discontinuity in both $\mu$ and $\tau_{\mu}$. The magnitude of the Weiss temperature is a little under three times that of the Neel temperature, indicating that the antiferromagnetically ordered state is established by interactions that extend beyond just the effective fields of the nearest neighbors, to the second nearest neighbor and potentially beyond. $^{36}$

The magnetic susceptibility data as a function of temperature of the 0.8 at.\% of Ni-doped BZT can be fit to the Curie-Weiss law (Equation 6) with a Weiss temperature, $\Theta$, of $-1.12 \mathrm{~K}$, as shown in Figure 4B. Since the Weiss temperature is non-zero and we did not observe evidence for a phase transition during our measurements, this suggests that this level of doping will be antiferromagnetic at a temperature below the lowest temperature measured. In contrast, the undoped BZT magnetic susceptibility data

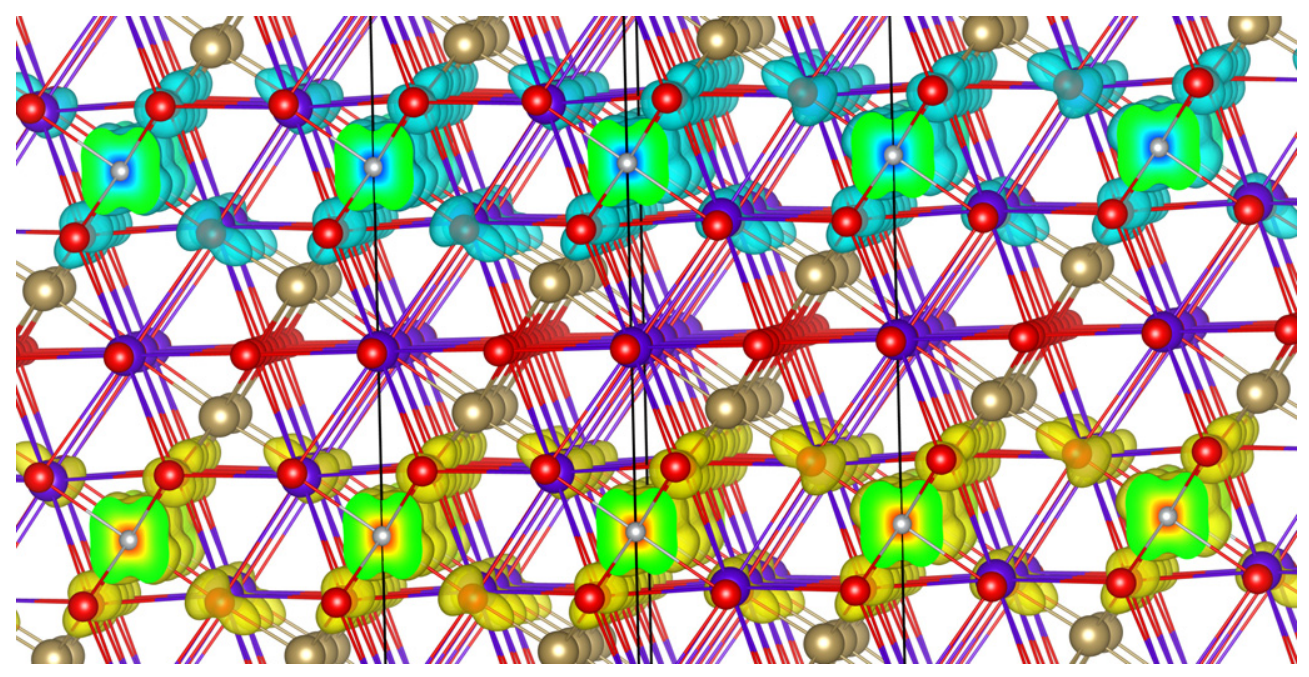

FIGURE 5 The magnetization charge density plot of antiferromagnetic structure of BNT crystal. The Ba, Ta, Ni, and O atoms are shown in purple, gold, gray, and red, respectively. The majority and the minority magnetization densities are shown in yellow and blue, respectively. The isosurface value is set to $0.003 \mathrm{e} / \AA^{3}$ [Color figure can be viewed at wileyonlinelibrary.com]
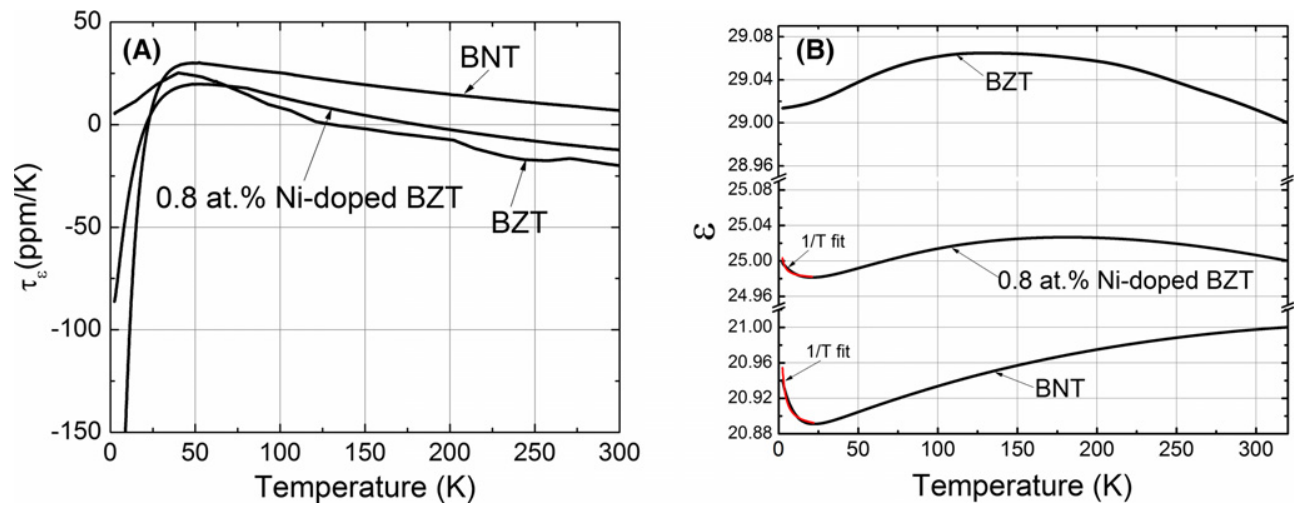

FIGURE 6 (A) The temperature coefficients of the dielectric constant $\left(\tau_{\varepsilon}\right)$ for BZT, BNT, and 0.8 at. $\%$ Ni-BZT are inferred from $\tau_{f}$, $\alpha_{\mathrm{L}}$, and $\tau_{\mu}$; (B) the dielectric constant $(\varepsilon)$ is obtained by integrating the $\tau_{\varepsilon}$ and then multiplying the normalized dielectric constants with the $\varepsilon_{0}$ of 29 , 21 , and 25 for BZT, BNT, and 0.8 at.\% Ni-BZT, respectively. The red solid lines at low temperatures are fits to the Curie law which shows reasonable agreement. [Color figure can be viewed at wileyonlinelibrary.com] 
$1514 \mid \bigcup_{\substack{x \in \text { American } \\ \text { Ceramic Society }}} \mathrm{O}_{\text {A }}$

(A)

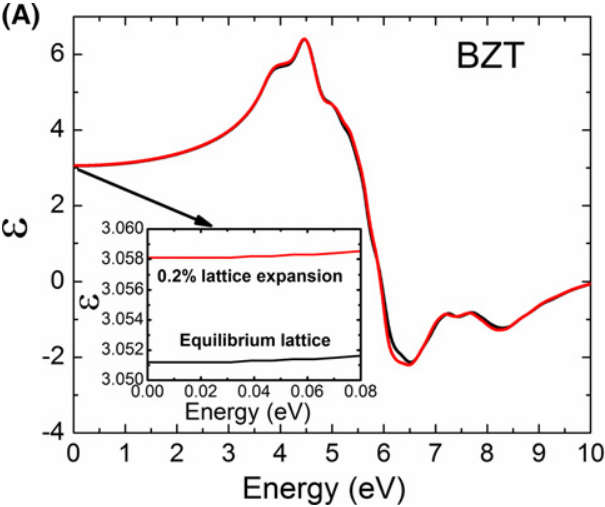

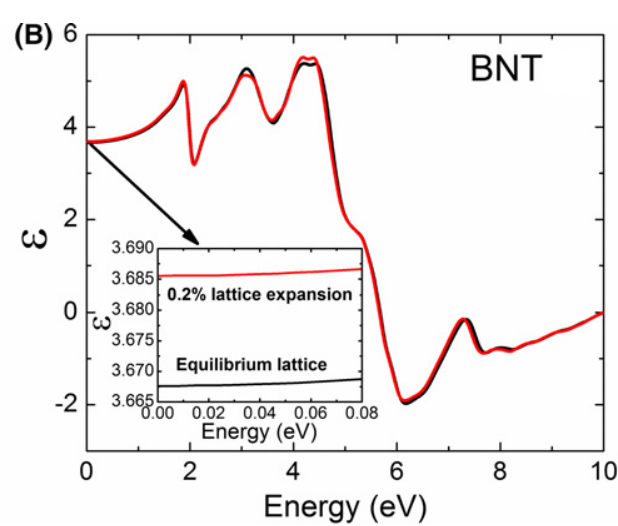

F I G URE 7 The electronic contribution to the dielectric constant of BZT (A) and BNT (B) with relaxed lattice and $0.2 \%$ of lattice expansion from density functional theory calculations [Color figure can be viewed at wileyonlinelibrary.com]

were found to be diamagnetic from 2 to $300 \mathrm{~K}$ and thus it does not fit the Curie law in this temperature range.

DFT calculations show that in BNT the ground-state antiferromagnetic configuration at $0 \mathrm{~K}$ exists in an intralayer-ferromagnetic and interlayer-antiferromagnetic structure as shown in Figure 5. Figure 5 shows that the magnetization density is not only localized on the $\mathrm{Ni}$ atoms. The nearest-neighbor $\mathrm{O}$ atoms of $\mathrm{Ni}$ atoms also carry magnetization and that is why the structure is intralayer-ferromagnetic. This configuration is $3.2 \mathrm{meV}$ per cell lower in energy than the ferromagnetic configuration.

\section{3 $\mid$ Determining $\tau_{\varepsilon}$}

To obtain the last unknown physical parameter, $\tau_{\varepsilon}$, the temperature dependence of the dielectric constant $\left(\tau_{\varepsilon}\right)$ can be inferred from $\tau_{\varepsilon}=-\left(2 \tau_{\mathrm{f}}+2 \alpha_{\mathrm{L}}+\tau_{\mu}\right)$, as shown in Figure 6A. The red solid lines in Figure 6B show that from 2 to $20 \mathrm{~K}$, the magnitude of $\varepsilon$ of 0.8 at.\% Ni-BZT and BNT roughly scales with the $\mathrm{Ni}$ content and can be reasonably fit with a Curie dependence with equation of $\varepsilon_{\infty}+{ }^{C} / T$, where $\varepsilon_{\infty}$ is the baseline dielectric constant before it changed its slope, a relation that is found for paraelectric materials or materials with paraelectric defects. ${ }^{37,39-41}$ Since the DFT calculations of the phonon characteristics do not show any indication of ferroelectricity in the materials studied, this observation must arise from the presence of paraelectric defects associated with $\mathrm{Ni}$. This can result from reorientation over an energy barrier of an asymmetric (1) $\mathrm{Ni}$ atom in the lattice, (2) spectator defect around the $\mathrm{Ni}$, or (3) d-shell electronic state. This is a surprising result and warrants further study.

We will now show why the temperature dependence of dielectric constant in BNT is different from the pure BZT compound, and how $\mathrm{Ni}$ influences the phonon spectrum and its associated dielectric constant shift with temperatures. As can be determined by the DFT calculations of the electronic contribution to $\varepsilon_{\mathrm{r}}$ in Figure 7, BZT's electronic contribution to the microwave dielectric constant (i.e., frequencies of $\sim 0.5-100 \mathrm{GHz}$ ) increases by 0.007 when it undergoes a $0.2 \%$ lattice expansion. For BNT, $\varepsilon_{\mathrm{r}}$ is increased by $\sim 0.02$ from 3.667 to 3.685 . Thus, the changes in the electronic polarizability accounts for $\sim 70 \%(0.01)$ and $33 \%$ (0.06) of the shift associated with $\varepsilon_{\mathrm{r}}$ of BZT and BNT over this range.

In future studies, we hope to extend current DFT methods of calculating the phonon contribution to the dielectric constant ${ }^{42}$ to model its temperature dependence.

\section{4 | CONCLUSION}

In this study, we used experimental methods to determine $\tau_{\mathrm{f}}, \tau_{\varepsilon,} \tau_{\mu}$, and $\alpha_{\mathrm{L}}$ over the temperature range of $2-300 \mathrm{~K}$ for BZT, 0.8 at.\% Ni-doped BZT, and BNT. These results, in combination with DFT calculations, have allowed us to develop a much improved understanding of the fundamental mechanisms responsible for determining $\tau_{\mathrm{f}}$ for these high-performance microwave dielectrics. From our work, we concluded:

1 A Grüneisen analysis using the Debye specific heat model can quantitatively fit the thermal expansion data over the entire temperature range investigated.

2 From 2 to $300 \mathrm{~K}, 70 \%$ of the observed temperature coefficient of resonant frequency $\left(\tau_{\mathrm{f}}\right)$ arises from the electroni contribution to the dielectric constant in BZT, in contrast to BNT where virtually the same fraction arises from the phonon contribution to the dielectric constant.

3 At temperatures below $25 \mathrm{~K}$, a Curie dependence from paraelectric defects dominates the temperature coefficient of resonant frequency $\left(\tau_{\mathrm{f}}\right)$.

4 The magnetic contribution to the temperature coefficient of resonant frequency $\left(\tau_{\mathrm{f}}\right)$ in Ni-containing material arises from spin excitations, but is over an order of magnitude smaller than the other contributions over all temperatures measured. 


\section{REFERENCES}

1. Sebastian MT. Dielectric Materials for Wireless Communication. Linacre House, Oxford, UK: Elsevier; 2008.

2. Reaney IM, Iddles D. Microwave dielectric ceramics for resonators and filters in mobile phone networks. $J$ Am Ceram Soc. 2006;89:2063-2072.

3. Kajfez D, Guillon P. Dielectric Resonators. Norwood, MA: Artech House; 1986.

4. Ning PF, Li LX, Zhang P, Xia WS. Raman scattering, electronic structure and microwave dielectric properties of $\mathrm{Ba}\left(\left[\mathrm{Mg}_{1-\mathrm{x}} \mathrm{Zn}_{\mathrm{x}}\right]_{1 /}\right.$ $\left.{ }_{3} \mathrm{Ta}_{2 / 3}\right) \mathrm{O}_{3}$ ceramics. Ceram Int. 2012;38:1391-1398.

5. Davies PK, Tong J, Negas T. Effect of ordering-induced domain boundaries on low loss $\mathrm{Ba}\left(\mathrm{Zn}_{1 / 3} \mathrm{Ta}_{2 / 3}\right) \mathrm{O}_{3}-\mathrm{BaZrO}_{3}$ perovskite microwave dielectrics. J Am Ceram Soc. 1997;80():1727-1740.

6. Negas T, Yeager G, Bell S, Amren R, Chemistry of Electronic Ceramic Materialsin Proceedings of the International Conference, Jackson, WY, July 17-20; 1990: 21-37 (National Institute of Standards and Technology Special Publications, Gaithersburg, MD, 804, 1991).

7. Endo K, Fujimoto K, Murakawa K. Dielectric properties of ceramics in $\mathrm{Ba}\left(\mathrm{Co}_{1 / 3} \mathrm{Nb}_{2 / 3}\right) \mathrm{O}_{3}-\mathrm{Ba}\left(\mathrm{Zn}_{1 / 3} \mathrm{Nb}_{2 / 3}\right) \mathrm{O}_{3}$ solid solutions. $J$ Am Ceram Soc. 1987;70():215-218.

8. Rong G, Newman N, Shaw B, Cronin D. Role of Ni and Zr doping on the electrical, optical, magnetic, and structural properties of barium zinc tantalate ceramics. J Mater Res. 1999;14:40114019.

9. Liu L, Matusevich A, Garg C, Newman N. The dominance of paramagnetic loss in microwave dielectric ceramics at cryogenic temperatures. Appl Phys Lett. 2012;101:252901-252905.

10. Liu L, Flores M, Newman N. Microwave loss in high-performance dielectric $\mathrm{Ba}\left(\mathrm{Zn}_{1 / 3} \mathrm{Ta}_{2 / 3}\right) \mathrm{O}_{3}$ at $4.2 \mathrm{~K}$. Phys Rev Lett. 2012;109:257601.

11. Data Sheet 8700 Series: Temperature Stable Resonators, No. 202894A, Trans-Tech Inc., 2013.

12. Matsumoto $\mathrm{H}$, Tamura $\mathrm{H}$, Wakino $\mathrm{K}$. $\mathrm{Ba}(\mathrm{Mg}, \mathrm{Ta}) \mathrm{O}_{3}-\mathrm{BaSnO}_{3}$ high-Q dielectric resonator. Jpn J Appl Phys. 1991;30:23472349.

13. Gvasaliya SN, Roessli B, Sheptyakov D, Lushnikov SG, Shaplygina TA. Neutron scattering study of $\mathrm{PbMg}_{1 / 3} \mathrm{Ta}_{2 / 3} \mathrm{O}_{3}$ and $\mathrm{BaMg}_{1 / 3} \mathrm{Ta}_{2 / 3} \mathrm{O}_{3}$ complex perovskites. Eur Phys $J$ B. 2004;40:235-241.

14. Wersing W. High frequency ceramics dielectric and their application for microwave components. In: Steele BCH, ed. Electronic Ceramics. Elsevier Science Publishers, New York, NY; 1991:6793.

15. Akbarzadeh AR, Kornev I, Malibert C, Bellaiche L, Kiat JM. Combined theoretical and experimental study of the low-temperature properties of $\mathrm{BaZrO}_{3}$ ". Phys Rev B. 2005;72:205104.

16. Bhuyan RK, Santhosh Kumar T, Pamu D, Renehan JM, Jacob $\mathrm{MV}$. Low temperature and broadband dielectric properties of $\mathrm{V}_{2} \mathrm{O}_{5}$ doped $\mathrm{Mg}_{2} \mathrm{TiO}_{4}$ ceramics. Mater Express. 2014;4:349358 .

17. Jacob MV, Pamu D, James Raju KC. Cryogenic microwave dielectric properties of sintered $\left(\mathrm{Zr}_{0.8} \mathrm{Sn}_{0.2}\right) \mathrm{TiO}_{4}$ doped with $\mathrm{CuO}$ and ZnO. J Am Ceram Soc. 2007;90():1511-1514.

18. Sudheendran K, Raju KCJ, Jacob MV. Microwave dielectric properties of Ti-substituted $\mathrm{Bi}_{2}\left(\mathrm{Zn}_{2 / 3} \mathrm{Nb}_{4 / 3}\right) \mathrm{O}_{7}$ pyrochlores at cryogenic temperatures. J Am Ceram Soc. 2009;92():1268-1271.
19. Subodh G, Ratheesh R, Jacob MV, Sebastian MT. Microwave dielectric properties and vibrational spectroscopic analysis of $\mathrm{MgTe}_{2} \mathrm{O}_{5}$ ceramics. J Mater Res. 2008;23():1551-1556.

20. Shimada $T$, Ichikawa $K$, Minemura $T$, et al. Intrinsic microwave dielectric loss of lanthanum aluminate. IEEE Trans Ultrason Ferroelect Freq Control. 2010;57:2243-2249.

21. Pamu D, Narayana Rao GL, James Raju KC, Jacob MV. Effect of $\mathrm{CuO}$ on the sintering and cryogenic microwave characteristics of $\left(\mathrm{Zr}_{0.8} \mathrm{Sn}_{0.2}\right) \mathrm{TiO}_{4}$ ceramics. Sci Tech Adv Mater. 2007;8:469476.

22. Varma MR, Kataria ND. Effect of dopants on the low temperature microwave dielectric properties of $\mathrm{Ba}\left(\mathrm{Zn}_{1 / 3} \mathrm{Ta}_{2 / 3}\right) \mathrm{O}_{3}$ ceramics. J Mater Sci: Mater Electron. 2007;18:441-446.

23. Zhang S, Devonport A, Newman N. Main source of microwave loss on transition-metal-doped $\mathrm{Ba}\left(\mathrm{Zn}_{1 / 3} \mathrm{Ta}_{2 / 3}\right) \mathrm{O}_{3}$ and $\mathrm{Ba}\left(\mathrm{Zn}_{1 / 3} \mathrm{Nb}_{2 /}\right.$ $\left.{ }_{3}\right)_{3}$ at cryogenic temperatures. J Am Ceram Soc. 2015;98 ():1188-1194.

24. Zhang S, Mechanisms responsible for microwave properties in high performance dielectric materials, Ph.D. Dissertation, Tempe, AZ: Arizona State University; 2016.

25. Tamura H, Matsumoto H, Wakino K. Low temperature properties of microwave dielectrics. Jpn J Appl Phys. 1989;28(Suppl. 28-2): 21-23.

26. Chen LF, Ong CK, Neo CP, Varadan VV, Varadan VK. Microwave Electronics: Measurement and Materials Characterization. West Sussex, UK: John Wiley and Sons; 2004.

27. Keysight literature No. 5991-2171EN, Measuring dielectric properties using keysight's materials measurement solutions. Keysight Technologies, Inc., 2016.

28. Application Note 1369-1, Agilent solutions for measuring permittivity and permeability with LCR meters and impedance analyzers, Agilent literature No. 5980-2862EN, Agilent Technologies, Inc., 2014.

29. Blöchl PE. Projector augmented-wave method. Phys Rev B. 1994;50:17953-17979.

30. Kresse G, Hafner J. Ab initio molecular dynamics for liquid metals. Phys Rev B. 1993;47:558-561.

31. Kresse G, Furthmüller J. Efficient iterative schemes for ab initio total-energy calculations using a plane-wave basis set. Phys Rev B. 1996;54:11169-11186.

32. Kresse G, Joubert J. From ultrasoft pseudopotentials to the projector augmented-wave method. Phys Rev B. 1999;59:1758-1775.

33. Perdew JP, Burke K, Ernzerhof M. Generalized gradient approximation made simple. Phys Rev Lett. 1996;77:3865-3868.

34. Alfe D. PHON: a program to calculate phonons using the small displacement method. Comp Phys Commun. 2009;180:26222633.

35. Willemsen HW, Armstrong RL, Meincke PPM. Thermal expansion measurements near the antiferromagnetic phase transitions in $\mathrm{K}_{2} \mathrm{ReCl}_{6}$ and $\mathrm{K}_{2} \mathrm{IrCl}_{6}$. J Low Temp Phys. 1977;26:299-309.

36. Ashcroft NW, Mermin ND. Solid State Physics. New York, NY: Holt, Rinehart and Winston; 1976.

37. Lowndes RP, Martin DH. Dielectric constants of ionic crystals and their variations with temperature and pressure. Proc Roy Lond A. 1970;316:351-375.

38. Shannon RD, Prewitt CT. Effective ionic radii in oxides and fluorides. Acta Cryst. 1969;B25:925-946.

39. Fiory AT. Electric dipole interaction among polar defects in alkali halides. Phys Rev B. 1971;4:614-627. 
40. Kanzig W, Hart HR, Roberts S. Paraelectricity and ferroelectricity due to hydroxyl ions in alali halides; paraelectric cooling. Phys Rev Lett. 1964;13:543-545.

41. Barrett JH. Dielectric constant in perovskite type crystal. Phys Rev. 1952;86():118-120.

42. Dai J-Q, Zhang H, Song Y-M. First-principles investigation of intrinsic dielectric response in $\mathrm{Ba}\left(\mathrm{B}^{\prime}{ }_{1 / 3} \mathrm{~B}^{\prime \prime}{ }_{2 / 3}\right) \mathrm{O}_{3}$ with $\mathrm{B}^{\prime}$ as transition metal cations. Mater Chem Phys. 2015;159:6-9.
How to cite this article: Zhang $\mathrm{S}$, Sahin $\mathrm{H}$, Torun E, et al. Fundamental mechanisms responsible for the temperature coefficient of resonant frequency in microwave dielectric ceramics. J Am Ceram Soc. 2017;100:1508-1516. doi: 10.1111/jace.14648. 